\title{
Impact of Skeletal Muscle Mass on Metabolic Health
}

\author{
Gyuri Kim, Jae Hyeon Kim
}

Division of Endocrinology and Metabolism, Department of Medicine, Samsung Medical Center, Sungkyunkwan University School of Medicine, Seoul, Korea

Skeletal muscle is regarded as an endocrine and paracrine organ. Muscle-derived secretory proteins, referred to as myokines, mediate interactions between skeletal muscle mass and other organs such as the liver, adipose tissue, pancreas, bone, and the cardiovascular system. As individuals age, reduced levels of physical activity and sarcopenia (loss of skeletal muscle mass and strength) are associated with physical frailty and disability. Recently, several studies have suggested that the loss of skeletal muscle mass may contribute to metabolic disease. Therefore, herein, we focus on the relationships between skeletal muscle mass and metabolic diseases, including metabolic syndrome and non-alcoholic fatty liver disease.

Keywords: Muscle, skeletal; Sarcopenia; Metabolic syndrome; Non-alcoholic fatty liver disease

The Namgok Award is the highest scientific award of the Korean Endocrine Society, and is given to honor an individual who has made excellent contributions to progress in the field of endocrinology and metabolism. The Namgok Award is named after the pen name of Professor Hun Ki Min, who founded the Korean Endocrine Society in 1982. Professor Jae Hyeon Kim received the Namgok Award at the Autumn Symposium of the Korean Endocrine Society in November 2019.

\section{INTRODUCTION}

Sarcopenia is a condition characterized by a progressive and generalized loss of skeletal muscle mass, strength, and/or physical performance [1]. The overall prevalence of sarcopenia among Korean women over the age of 50 years is estimated as $20.2 \%$ [2]. In recent years, researchers have come to regard sarcopenia not only as a part of aging, but also as a progressive disease associated with metabolic disorders such as metabolic syndrome, diabetes, non-alcoholic fatty liver disease (NAFLD), and cardiovascular disease. Several cross-sectional and longitudinal studies have reported that sarcopenia is a novel risk factor for the development of metabolic diseases. Furthermore, the pathophysiology of sarcopenia and metabolic diseases is complex, involving several processes, and has not been completely elucidated. Herein, we review the possible mechanisms underlying sarcopenia and metabolic diseases.

\section{MECHANISMS RELATED TO SARCOPENIA AND METABOLIC DISEASE}

\section{Insulin resistance}

Skeletal muscle is the major organ in which insulin-mediated glucose uptake by glucose transporter 4 (GLUT4) takes place. A previous report showed that muscle-specific knockout of GLUT4 resulted in severe insulin resistance and impaired glu-
Received: 3 March 2020, Revised: 5 March 2020, Accepted: 10 March 2020 Corresponding author: Jae Hyeon Kim

Division of Endocrinology and Metabolism, Department of Medicine, Samsung Medical Center, Sungkyunkwan University School of Medicine, 81 Irwon-ro, Gangnam-gu, Seoul 06351, Korea

Tel: +82-2-3410-1580, Fax:+82-2-3410-3849, E-mail: jaehyeon@skku.edu
Copyright (C) 2020 Korean Endocrine Society

This is an Open Access article distributed under the terms of the Creative Commons Attribution Non-Commercial License (https://creativecommons.org/ licenses/by-nc/4.0/) which permits unrestricted non-commercial use, distribution, and reproduction in any medium, provided the original work is properly cited. 
cose tolerance [3]. Insulin resistance resulting from loss of skeletal muscle mass increases lipolysis and the consequent release of free fatty acids from adipose tissue, which leads to inhibition of the growth hormone/insulin growth factor-1 axis and reduced muscle regeneration [4]. Insulin resistance leads to promotion of gluconeogenesis, upregulation of sterol regulatory elementbinding protein 1c (SREBP-1c), inhibition of $\beta$-oxidation, increased free fatty acid delivery, and altered triglyceride transport, which causes triglycerides to accumulate in skeletal muscle and the liver [5]. In compensatory hyperinsulinemia, which is induced by insulin resistance, impaired suppression of gluconeogenesis promotes proteolysis, reduces protein synthesis; for this reason, compensatory hyperinsulinemia may be involved in age-related muscle loss and sarcopenia [6,7].

\section{Myokines}

Skeletal muscle is a metabolically active organ that interacts with other organs through secretory proteins, including cytokines and peptides, to mediate energy metabolism and exert beneficial effects on metabolic health [8]. These proteins, known as myokines, are secreted in response to muscle contraction or strength training [9]. Regular physical activity and exercise promote increased skeletal muscle mass and myokine release [10]. Myokines including interleukin 6 (IL-6), myostatin, myonectin, and irisin affect adipogenesis in paracrine and endocrine fashions. Intramuscular adipose tissue seems to be an important target tissue for paracrine myokines such as musclin, which is induced during exercise and promotes peroxisome proliferator-activated receptor- $\alpha$ (PPAR- $\alpha$ )-dependent fat browning $[11,12]$. IL-6 is an important exercise-inducible myokine that enhances glucose production in the liver, lipolysis in adipose tissue, pancreatic $\beta$-cell viability, and insulin secretion [13-15]. Intramuscular IL-6 promotes glucose uptake and fat oxidation via the phosphoinositide 3-kinase (PI3K) and AMP-activated protein kinase (AMPK) signaling pathways, respectively, and improves insulin sensitivity by blocking proinflammatory signaling pathways in the muscle $[16,17]$. In inflammation-prone animal models, elevation of hepatic IL-6 downregulates lipogenic genes, but upregulates fatty acid oxidation-related genes in the liver, thereby exerting a protective effect on the development of hepatic steatosis [18]. However, IL-6 released by adipose tissue and its chronic elevation in response to a high-fat diet can promote obesity by recruiting macrophages into adipose tissue [19]. Irisin, a myokine induced by exercise, also increases energy expenditure through PPAR- $\alpha$-dependent downsignaling and improves insulin sensitivity and hepatic steatosis by upregulating the fibroblast growth factor 21 (FGF21); these effects were observed independently of reduction in body weight and adiposity in an diet-induced obese mouse model [20,21]. In human muscle, expression of the fibronectin type III domain-containing protein 5 (FNDC5) gene, which encodes a precursor of irisin, is positively associated with physical activity and skeletal muscle mass [22]. Therefore, the presence of a relatively large quantity of skeletal muscle mass may result in the secretion of high levels of favorable myokines, resulting in beneficial effects on glucose uptake and lipid metabolism.

\section{Chronic inflammation}

Sarcopenia is linked with chronic inflammation. In patients with sarcopenia, elevated levels of C-reactive protein (CRP) and proinflammatory cytokines were found, as well as decreased levels of anti-inflammatory cytokines, reflecting conditions of chronic inflammation. Levels of CRP and IL-6 were negatively associated with appendicular lean body mass $[23,24]$. Furthermore, intramuscular lipid accumulation also impairs mitochondrial function by inhibiting the capacity of mitochondria to carry out $\beta$-oxidation and increasing the formation of reactive oxygen species, accompanied by increased secretion of pro-inflammatory cytokines such as tissue necrosis factor (TNF)- $\alpha$, IL-6, leptin, and resistin, which contribute to the development and aggravation of systemic insulin resistance [25]. Increased TNF- $\alpha$ levels in the liver cause lipid accumulation by activating de novo fat synthesis [26]. TNF- $\alpha$ also directly or indirectly activates nuclear factor kappa $\mathrm{B}$, a major transcription factor that promotes the expression of numerous proinflammatory cytokines, which contribute to skeletal muscle catabolism and the development of NAFLD [27].

\section{Vitamin D}

Vitamin D modulates the physiology and function of multiple organ systems in humans, and its deficiency is a common health problem that occurs with aging [28]. Previous studies have reported that low levels of vitamin D are associated with sarcopenia and that vitamin D may play an important role in the growth of skeletal muscle mass and maintenance of muscle function $[29,30]$. The nuclear vitamin D receptor has been identified in human skeletal muscle, and the combination of its decreased expression with aging and vitamin D deficiency may lead to sarcopenia; as a result, individuals with sarcopenia may show lower vitamin $\mathrm{D}$ levels independently from the effects of metabolic syndrome, diabetes, and insulin resistance [31]. Vitamin D upregulates PPAR- $\alpha$ and carnitine palmitoyltransferase-1, 
which are involved in hepatic fatty acid oxidation, while it downregulates the expression of SREBP-1c, acetyl coenzyme A carboxylase, and fatty acid synthase, which mediate lipogenesis [32]. Vitamin D deficiency exacerbates NAFLD through the activation of Toll-like receptors, and it causes hepatic insulin resistance and the upregulation of genes linked to hepatic inflammation and oxidative stress [33].

\section{Physical inactivity}

Physical inactivity exerts a catabolic effect in muscle tissue because amino acids lose their ability to stimulate protein synthesis [34]. Furthermore, muscle disuse-induced atrophy involves a decrease in energy consumption that causes increased fat deposition, systemic inflammation, and insulin resistance [35]. Numerous myokines are secreted during exercise, and physical activity results in decreased pro-inflammatory and increased antiinflammatory cytokine production, muscle hypertrophy, regeneration, and increased glucose uptake; through these mechanisms, exercise decreases hepatic steatosis even without weight loss [36].

\section{SKELETAL MUSCLE MASS AND METABOLIC DISEASES}

\section{Metabolic syndrome}

Metabolic syndrome is a cluster of pathological metabolic conditions that includes abdominal obesity, insulin resistance, hyperglycemia, dyslipidemia, and hypertension [37]. In 1990, 50 million Americans were estimated to have metabolic syndrome, which increased to 64 million in 2000 [38]. In Korea, the prevalence of metabolic syndrome increased significantly from $24.9 \%$ in 1998 to $31.3 \%$ in 2007 [39]. Metabolic syndrome is a global health problem, as it is closely associated with cardiovascular disease, diabetes, cancer, and mortality [40]. The loss of skeletal muscle and the accumulation of intramuscular fat, which induce contractile impairment and metabolic abnormalities, may be associated with metabolic syndrome via a complex set of factors including proinflammatory cytokines, oxidative stress, mitochondrial dysfunction, insulin resistance, and lack of physical activity [41]. Several studies have established links between sarcopenia and metabolic syndrome.

A meta-analysis of 13 cross-sectional studies with 35,581 middle-aged and older non-obese adults showed that the overall prevalence of metabolic syndrome was $36.45 \%$ ( $95 \%$ confidence interval [CI], $28.28 \%$ to $45.48 \%$ ) in individuals with sarcopenia, and a positive association was found between sarcope- nia and metabolic syndrome (odds ratio [OR], 2.01; 95\% CI, 1.63 to 2.47) [42]. A longitudinal study of a hospital-based cohort comprising 1,042 Korean individuals reported that the incidence of metabolic syndrome over the course of 2 years was $10.5 \%$. They also found the percent of skeletal muscle mass relative to body weight was negatively associated with high waist circumference (OR, 0.7; 95\% CI, 0.50 to 0.99 ), high blood pressure (OR, $0.72 ; 95 \% \mathrm{CI}, 0.61$ to 0.86 ), and high triglycerides (OR, $0.77 ; 95 \% \mathrm{CI}, 0.61$ to 0.97 ), while no significant association was found with dysglycemia or low high-density lipoprotein cholesterol [43]. Furthermore, we conducted a longitudinal study investigating the association of low muscle mass and metabolic syndrome in 14,830 individuals examined at a health promotion center [44]. Skeletal muscle mass was measured in terms of the skeletal muscle mass index (SMI), a value representing body weight-adjusted appendicular skeletal muscle mass, which was estimated by bioelectrical impedance analysis. During 7 years of follow-up, $20.1 \%$ of the subjects developed metabolic syndrome. The highest sex-specific SMI tertile showed a significant inverse association with the development of metabolic syndrome (adjusted hazard ratio [HR], 0.61; 95\% CI, 0.54 to 0.68$)$ compared with the lowest tertile at baseline. In addition, compared with SMI changes of $<0 \%$ over a year, $>1 \%$ changes in SMI over a year were associated with a significantly reduced risk of developing metabolic syndrome even after adjustment for baseline SMI and glycometabolic parameters (adjusted HR, $0.67 ; 95 \% \mathrm{CI}, 0.56$ to 0.79 ). The data showed that low relative skeletal muscle mass is a causative factor for developing metabolic syndrome and an increase in relative skeletal muscle mass over time may have a preventive effect against the development of metabolic syndrome.

\section{Non-alcoholic fatty liver disease}

From a global perspective, NAFLD is the most rapidly growing metabolic liver disease, and it is receiving greater attention from researchers in parallel with the dramatic increase in the prevalence of obesity and insulin resistance [45]. The worldwide prevalence of NAFLD is estimated to be $25 \%$, and NAFLD imposes a large socioeconomic burden globally [46]. NAFLD encompasses a broad spectrum of metabolic liver disease, ranging from simple steatosis to non-alcoholic steatohepatitis (NASH), and it can progress to advanced liver disease and hepatocellular carcinoma; however, the most common outcome of NAFLD is related to cardiometabolic disease [47]. Several studies have investigated the relationship between sarcopenia and NAFLD.

A previous meta-analysis of five cross-sectional studies re- 
ported that patients with sarcopenia had a 1.5-fold increased risk of NAFLD compared to those without sarcopenia (OR, 1.54; $95 \%$ CI, 1.05 to 2.26) [48]. Another meta-analysis of six crosssectional studies with a total of 19,024 subjects showed that subjects with sarcopenia had a 1.3-fold increased risk of NAFLD (OR, 1.29; 95\% CI, 1.12 to 1.49) compared to those without sarcopenia [49]. Additionally, a meta-analysis of three studies with 3,226 subjects found that sarcopenia was associated with NASH (OR, 2.35; 95\% CI, 1.45 to 3.81) and advanced liver fibrosis (OR, 2.41; 95\% CI, 1.94 to 2.98) [50]. We conducted a longitudinal study regarding the association between low muscle mass and NAFLD in a general population cohort of 12,624 subjects without baseline NAFLD and 2,943 subjects with baseline NAFLD [51]. NAFLD was diagnosed by the hepatic steatosis index. The highest sex-specific SMI tertile was inversely associated with the development of NAFLD (adjusted HR, 0.44 ; $95 \%$ CI, 0.38 to 0.51 ) and positively associated with the resolution of NAFLD at baseline (adjusted HR, 2.09; 95\% CI, 1.02 to 4.28 ) compared with the lowest tertile at baseline. In addition, compared with the lowest tertile of change in SMI over a year, the highest tertile showed a significantly reduced risk of incident NAFLD (adjusted HR, 0.69; 95\% CI, 0.59 to 0.82 ) and a higher likelihood of resolution of baseline NAFLD (adjusted HR, 4.17; 95\% CI, 1.90 to 6.17) even after adjustment for baseline SMI. This study demonstrated that low relative skeletal muscle mass is a causative factor in the development of NAFLD. As no drugs have been approved by the Food and Drug Administration for the treatment of NAFLD, building relative skeletal muscle mass may emerge as a promising therapeutic modality for NAFLD.

\section{CONCLUSIONS}

Due to the aging of society, sarcopenia - a condition characterized by progressive and generalized loss of skeletal muscle mass and strength that places individuals at risk for physical disability, poor quality of life, and death — has been drawing increased attention. Many studies have linked the common mechanisms underlying sarcopenia and metabolic disease, including insulin resistance and obesity, and recently, low relative skeletal muscle mass and its decline over time have been established as causative risk factors for metabolic syndrome and NAFLD. Further studies are warranted to elucidate the effects of interventions including physical exercise and nutritional supplementation on both sarcopenia and metabolic disease.

\section{CONFLICTS OF INTEREST}

No potential conflict of interest relevant to this article was reported.

\section{ACKNOWLEDGMENTS}

I, Jae Hyeon Kim, would like to express my sincere gratitude to emeritus professor Hun Ki Min (Namgok) for giving me the prestigious Namgok Award. I would like to thank Prof. Kwangwon Kim, Prof. Moon-Kyu Lee, Prof. Myung-Shik Lee, Prof. Yong-Ki Min, Prof. Kyu Yeon Hur, Prof. Sang-Man Jin and Prof. Gyuri Kim at Samsung Medical Center, Sungkyunkwan University School of Medicine, and all the professor-mentors who guided and taught me. This work was supported by the Korean Endocrine Society through the 2019 Namgok Award.

\section{ORCID}

Gyuri Kim https://orcid.org/0000-0002-2242-2816

Jae Hyeon Kim https://orcid.org/0000-0001-5001-963X

\section{REFERENCES}

1. Cruz-Jentoft AJ, Bahat G, Bauer J, Boirie Y, Bruyere O, Cederholm T, et al. Sarcopenia: revised European consensus on definition and diagnosis. Age Ageing 2019;48:16-31.

2. Kwon HJ, Ha YC, Park HM. Prevalence of sarcopenia in the Korean woman based on the Korean National Health and Nutritional Examination Surveys. J Bone Metab 2016; 23:23-6.

3. Zisman A, Peroni OD, Abel ED, Michael MD, Mauvais-Jarvis F, Lowell BB, et al. Targeted disruption of the glucose transporter 4 selectively in muscle causes insulin resistance and glucose intolerance. Nat Med 2000;6:924-8.

4. Kalyani RR, Corriere M, Ferrucci L. Age-related and disease-related muscle loss: the effect of diabetes, obesity, and other diseases. Lancet Diabetes Endocrinol 2014;2:819-29.

5. Postic C, Girard J. Contribution of de novo fatty acid synthesis to hepatic steatosis and insulin resistance: lessons from genetically engineered mice. J Clin Invest 2008;118:829-38.

6. Bonaldo P, Sandri M. Cellular and molecular mechanisms of muscle atrophy. Dis Model Mech 2013;6:25-39.

7. Fujita S, Glynn EL, Timmerman KL, Rasmussen BB, Volpi E. Supraphysiological hyperinsulinaemia is necessary to stimulate skeletal muscle protein anabolism in older adults: 
evidence of a true age-related insulin resistance of muscle protein metabolism. Diabetologia 2009;52:1889-98.

8. Hartwig S, Raschke S, Knebel B, Scheler M, Irmler M, Passlack $\mathrm{W}$, et al. Secretome profiling of primary human skeletal muscle cells. Biochim Biophys Acta 2014;1844:1011-7.

9. Raschke S, Eckardt K, Bjorklund Holven K, Jensen J, Eckel J. Identification and validation of novel contraction-regulated myokines released from primary human skeletal muscle cells. PLoS One 2013;8:e62008.

10. Jeon YK, Kim SS, Kim JH, Kim HJ, Kim HJ, Park JJ, et al. Combined aerobic and resistance exercise training reduces circulating apolipoprotein $\mathrm{J}$ levels and improves insulin resistance in postmenopausal diabetic women. Diabetes Metab J 2020;44:103-12.

11. Komolka K, Albrecht E, Wimmers K, Michal JJ, Maak S. Molecular heterogeneities of adipose depots: potential effects on adipose-muscle cross-talk in humans, mice and farm animals. J Genomics 2014;2:31-44.

12. Jeremic N, Chaturvedi P, Tyagi SC. Browning of white fat: novel insight into factors, mechanisms, and therapeutics. J Cell Physiol 2017;232:61-8.

13. Pedersen BK, Febbraio MA. Muscle as an endocrine organ: focus on muscle-derived interleukin-6. Physiol Rev 2008;88: 1379-406.

14. Ellingsgaard H, Hauselmann I, Schuler B, Habib AM, Baggio LL, Meier DT, et al. Interleukin-6 enhances insulin secretion by increasing glucagon-like peptide-1 secretion from L cells and alpha cells. Nat Med 2011;17:1481-9.

15. Paula FM, Leite NC, Vanzela EC, Kurauti MA, Freitas-Dias $\mathrm{R}$, Carneiro EM, et al. Exercise increases pancreatic $\beta$-cell viability in a model of type 1 diabetes through IL-6 signaling. FASEB J 2015;29:1805-16.

16. Lambernd S, Taube A, Schober A, Platzbecker B, Gorgens SW, Schlich R, et al. Contractile activity of human skeletal muscle cells prevents insulin resistance by inhibiting pro-inflammatory signalling pathways. Diabetologia 2012;55: 1128-39.

17. Giudice J, Taylor JM. Muscle as a paracrine and endocrine organ. Curr Opin Pharmacol 2017;34:49-55.

18. Miller AM, Wang H, Bertola A, Park O, Horiguchi N, Ki $\mathrm{SH}$, et al. Inflammation-associated interleukin-6/signal transducer and activator of transcription 3 activation ameliorates alcoholic and nonalcoholic fatty liver diseases in interleukin-10-deficient mice. Hepatology 2011;54:846-56.

19. Kraakman MJ, Kammoun HL, Allen TL, Deswaerte V, Henstridge DC, Estevez E, et al. Blocking IL-6 trans-signaling prevents high-fat diet-induced adipose tissue macrophage recruitment but does not improve insulin resistance. Cell Metab 2015;21:403-16.

20. Zhang HJ, Zhang XF, Ma ZM, Pan LL, Chen Z, Han HW, et al. Irisin is inversely associated with intrahepatic triglyceride contents in obese adults. J Hepatol 2013;59:557-62.

21. Xu J, Lloyd DJ, Hale C, Stanislaus S, Chen M, Sivits G, et al. Fibroblast growth factor 21 reverses hepatic steatosis, increases energy expenditure, and improves insulin sensitivity in diet-induced obese mice. Diabetes 2009;58:250-9.

22. Kurdiova T, Balaz M, Vician M, Maderova D, Vlcek M, Valkovic L, et al. Effects of obesity, diabetes and exercise on Fndc5 gene expression and irisin release in human skeletal muscle and adipose tissue: in vivo and in vitro studies. J Physiol 2014;592:1091-107.

23. Hong HC, Hwang SY, Choi HY, Yoo HJ, Seo JA, Kim SG, et al. Relationship between sarcopenia and nonalcoholic fatty liver disease: the Korean Sarcopenic Obesity Study. Hepatology 2014;59:1772-8.

24. Cesari M, Pedone C, Incalzi RA, Pahor M. ACE-inhibition and physical function: results from the Trial of AngiotensinConverting Enzyme Inhibition and Novel Cardiovascular Risk Factors (TRAIN) study. J Am Med Dir Assoc 2010;11: 26-32.

25. Woo CY, Jang JE, Lee SE, Koh EH, Lee KU. Mitochondrial dysfunction in adipocytes as a primary cause of adipose tissue inflammation. Diabetes Metab J 2019;43:247-56.

26. Wree A, Kahraman A, Gerken G, Canbay A. Obesity affects the liver: the link between adipocytes and hepatocytes. Digestion 2011;83:124-33.

27. Beyer I, Mets T, Bautmans I. Chronic low-grade inflammation and age-related sarcopenia. Curr Opin Clin Nutr Metab Care 2012;15:12-22.

28. Remelli F, Vitali A, Zurlo A, Volpato S. Vitamin D deficiency and sarcopenia in older persons. Nutrients 2019;11:2861.

29. Scott D, Blizzard L, Fell J, Ding C, Winzenberg T, Jones G. A prospective study of the associations between 25-hydroxy-vitamin $\mathrm{D}$, sarcopenia progression and physical activity in older adults. Clin Endocrinol (Oxf) 2010;73:581-7.

30. Ceglia L. Vitamin D and skeletal muscle tissue and function. Mol Aspects Med 2008;29:407-14.

31. Barchetta I, Angelico F, Del Ben M, Baroni MG, Pozzilli P, Morini $\mathrm{S}$, et al. Strong association between non alcoholic fatty liver disease (NAFLD) and low 25(OH) vitamin D levels in an adult population with normal serum liver enzymes. BMC Med 2011;9:85. 
32. Yin Y, Yu Z, Xia M, Luo X, Lu X, Ling W. Vitamin D attenuates high fat diet-induced hepatic steatosis in rats by modulating lipid metabolism. Eur J Clin Invest 2012;42:1189-96.

33. Roth CL, Elfers CT, Figlewicz DP, Melhorn SJ, Morton GJ, Hoofnagle A, et al. Vitamin D deficiency in obese rats exacerbates nonalcoholic fatty liver disease and increases hepatic resistin and Toll-like receptor activation. Hepatology 2012; 55:1103-11.

34. Biolo G, Cederholm T, Muscaritoli M. Muscle contractile and metabolic dysfunction is a common feature of sarcopenia of aging and chronic diseases: from sarcopenic obesity to cachexia. Clin Nutr 2014;33:737-48.

35. Lang T, Streeper T, Cawthon P, Baldwin K, Taaffe DR, Harris TB. Sarcopenia: etiology, clinical consequences, intervention, and assessment. Osteoporos Int 2010;21:543-59.

36. Oh KJ, Lee DS, Kim WK, Han BS, Lee SC, Bae KH. Metabolic adaptation in obesity and type II diabetes: myokines, adipokines and hepatokines. Int J Mol Sci 2016;18:8.

37. Cornier MA, Dabelea D, Hernandez TL, Lindstrom RC, Steig AJ, Stob NR, et al. The metabolic syndrome. Endocr Rev 2008;29:777-822.

38. Grundy SM. Metabolic syndrome pandemic. Arterioscler Thromb Vasc Biol 2008;28:629-36.

39. Lim S, Shin H, Song JH, Kwak SH, Kang SM, Won Yoon J, et al. Increasing prevalence of metabolic syndrome in Korea: the Korean National Health and Nutrition Examination Survey for 1998-2007. Diabetes Care 2011;34:1323-8.

40. Lakka HM, Laaksonen DE, Lakka TA, Niskanen LK, Kumpusalo E, Tuomilehto J, et al. The metabolic syndrome and total and cardiovascular disease mortality in middle-aged men. JAMA 2002;288:2709-16.

41. Rubio-Ruiz ME, Guarner-Lans V, Perez-Torres I, Soto ME. Mechanisms underlying metabolic syndrome-related sarcopenia and possible therapeutic measures. Int J Mol Sci 2019; 20:647.

42. Zhang H, Lin S, Gao T, Zhong F, Cai J, Sun Y, et al. Associ- ation between sarcopenia and metabolic syndrome in middle-aged and older non-obese adults: a systematic review and meta-analysis. Nutrients 2018;10:364.

43. Park BS, Yoon JS. Relative skeletal muscle mass is associated with development of metabolic syndrome. Diabetes Metab J 2013;37:458-64.

44. Kim G, Lee SE, Jun JE, Lee YB, Ahn J, Bae JC, et al. Increase in relative skeletal muscle mass over time and its inverse association with metabolic syndrome development: a 7-year retrospective cohort study. Cardiovasc Diabetol 2018; 17:23.

45. Loomba R, Sanyal AJ. The global NAFLD epidemic. Nat Rev Gastroenterol Hepatol 2013;10:686-90.

46. Younossi ZM, Koenig AB, Abdelatif D, Fazel Y, Henry L, Wymer M. Global epidemiology of nonalcoholic fatty liver disease: meta-analytic assessment of prevalence, incidence, and outcomes. Hepatology 2016;64:73-84.

47. Rinella ME. Nonalcoholic fatty liver disease: a systematic review. JAMA 2015;313:2263-73.

48. Wijarnpreecha K, Panjawatanan P, Thongprayoon C, Jaruvongvanich V, Ungprasert P. Sarcopenia and risk of nonalcoholic fatty liver disease: a meta-analysis. Saudi J Gastroenterol 2018;24:12-7.

49. Pan X, Han Y, Zou T, Zhu G, Xu K, Zheng J, et al. Sarcopenia contributes to the progression of nonalcoholic fatty liver disease-related fibrosis: a meta-analysis. Dig Dis 2018;36: 427-36.

50. Yu R, Shi Q, Liu L, Chen L. Relationship of sarcopenia with steatohepatitis and advanced liver fibrosis in non-alcoholic fatty liver disease: a meta-analysis. BMC Gastroenterol 2018;18:51.

51. Kim G, Lee SE, Lee YB, Jun JE, Ahn J, Bae JC, et al. Relationship between relative skeletal muscle mass and nonalcoholic fatty liver disease: a 7-year longitudinal study. Hepatology 2018;68:1755-68. 